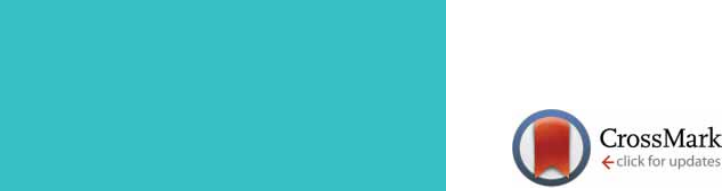

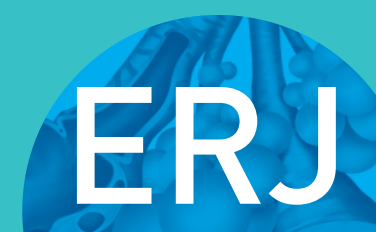

open research

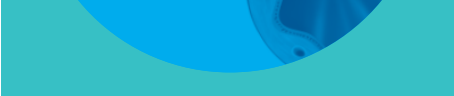

\section{Sex disparities in cystic fibrosis: review on the effect of female sex hormones on lung pathophysiology and outcomes}

\author{
Grace Y. Lam (1) ${ }^{1,2}$, Jodi Goodwin², Pearce G. Wilcox ${ }^{2}$ and Bradley S. Quon ${ }^{1,2}$ \\ Affiliations: ${ }^{1}$ Centre for Heart Lung Innovation, St Paul's Hospital, University of British Columbia, Vancouver, \\ BC, Canada. ${ }^{2}$ Adult Cystic Fibrosis Program, St Paul's Hospital, Vancouver, BC, Canada.
}

Correspondence: Grace Y. Lam, Centre for Heart Lung Innovation, St Paul's Hospital, University of British Columbia, 1081 Burrard Street Vancouver, BC V6Z 1Y6, Canada. E-mail: glamaualberta.ca

ABSTRACT Sex differences in morbidity and mortality have been reported in the cystic fibrosis (CF) population worldwide. However, it is unclear why CF women have worse clinical outcomes than men. In this review, we focus on the influence of female sex hormones on CF pulmonary outcomes and summarise data from in vitro and in vivo experiments on how oestrogen and progesterone might modify mucociliary clearance, immunity and infection in the CF airways. The potential for novel sex hormone-related therapeutic interventions is also discussed.

@ERSpublications

A disparity in survival has been noted between men and women with cystic fibrosis where female sex hormones may facilitate lung disease progression. There is strong evidence that implicates oestrogen in numerous aspects of CF airway pathophysiology. https://bit.ly/34ef $4 \mathrm{Cv}$

Cite this article as: Lam GY, Goodwin J, Wilcox PG, et al. Sex disparities in cystic fibrosis: review on the effect of female sex hormones on lung pathophysiology and outcomes. ERJ Open Res 2021; 7: 00475-2020 [https://doi.org/10.1183/23120541.00475-2020]. 


\section{Introduction}

Cystic fibrosis (CF) is a multi-system genetic disease due to defects in the CF transmembrane conductance regulator (CFTR) protein, most commonly affecting the lungs. This manifests as chronic airway infection and inflammation, and recurrent pulmonary exacerbations, which account for the majority of morbidity and mortality seen in CF. Other risk factors associated with mortality include certain CFTR genotypes, poor nutritional status, development of CF-related diabetes (CFRD), low socioeconomic status, minority race or ethnicity and female sex [1,2]. Indeed, researchers have identified a persistent disadvantage in clinical outcomes and survival for female CF patients compared to their male counterparts. This disparity in outcomes between sexes is not limited to CF as a number of other respiratory diseases, such as asthma, also have worse reported outcomes in women [3]. While there are some anatomical differences between males and females, such as smaller airway diameter and lung volumes in women, that might contribute to differences in respiratory disease outcomes [4], many have theorised that sex hormones may be contributing to differences in CFTR expression/function, mucus and airway fluid characteristics, as well as airway infection and inflammation. Here, we provide a focused review of the literature on the influence of oestrogen and progesterone on CF airway pathophysiology and pulmonary outcomes.

\section{The "gender gap" in CF survival and pulmonary outcomes}

The gap in clinical outcomes between men and women with CF has been well documented in epidemiological studies [5] and confirmed over time with registry data worldwide. The 2017 CF Canada registry reported a median survival age of 56 years for males and just 49 years for females [6]. Similarly, this discrepancy is seen in registry data from the USA, UK, Scandinavia and Australia where women also experience a higher rate of morbidity and mortality $[5,7-10]$.

Women demonstrate earlier or more frequent acquisition of organisms associated with worse outcomes including Pseudomonas aeruginosa, Stenotrophomonas maltophilia, methicillin-resistant Staphylococcus aureus (MRSA), Burkholderia species, fungal and non-tuberculous mycobacterial species [8, 9, 11]. Women also need more intense and complex pulmonary exacerbation treatment regimens and require longer lengths of hospitalisation $[8,9]$. As well, CFRD is a more common complication in women and they experience a larger drop in lung function and a greater mortality rate after developing CFRD than men $[12,13]$, though the cause for this remains unclear.

The cause of this "gender gap" is likely multifactorial, related to intrinsic physiological differences as well as biochemical, nutritional and behavioural differences between the sexes. Women, on average, have a smaller airway diameter with smaller lung volume, even adjusted for height, which has been speculated to represent an anatomical impediment to mucus clearance particularly from airways with sharp-angled bifurcations or trifurcations [3]. In addition, women are predisposed to a higher degree of airway hyperresponsiveness and bronchoconstriction $[3,4]$. This increased propensity for bronchoconstriction in physiologically smaller airways may result in more frequent and exaggerated narrowing of inflamed airways during periods of exacerbations, leading to more mucus production and impaction, thus perpetuating the vicious cycle of inflammation and recurrent bacterial infections. Furthermore, body mass index (BMI) has been well documented to be lower in CF women compared to $\mathrm{CF}$ men and is significantly lower than age-matched non-CF women starting at a younger age [14]. Given the reported desirability of having a lower weight, CF women are likely to be more resistant to nutritional interventions that help to increase their weight [15]. Vertical growth, another indicator of nutritional status, is also stunted in CF girls compared to age-matched non-CF girls, while the same disparity is not seen in CF boys [16]. Poor nutritional status and reduced BMI are associated with lower lung function and increased mortality in CF $[9,14,16]$, and therefore undoubtedly contribute to the sex disparity in CF survival. Furthermore, CF women have been found to have poorer medication and nutritional adherence, which may also contribute to reduced lung function [17, 18] and worse health outcomes [19].

Despite accounting for differences in BMI, lung function, CFRD status and chronic bacterial infections between the sexes, female sex remains an independent risk factor for death [9]. It has been noted that sex differences in outcomes seem to only manifest after puberty as rates of pulmonary exacerbations and mortality are similar between boys and girls before puberty but are more common in women than men in adulthood [20, 21]. Therefore, disparity in morbidity and mortality between sexes may also be related to post-pubertal female hormonal changes.

\section{Physiology of female sex hormones}

\section{Biology of female sex hormones}

The predominant female sex hormones are oestrogen and progesterone. Oestrogen is mainly produced from cholesterol by the ovaries, the corpus luteum and the placenta [22] but can also be produced in extra-gonadal sites including peripheral adipose tissue and the lungs via the enzyme aromatase. Physiological 
oestrogens exist in three biochemical forms: oestrone, oestradiol (or 17- $\beta$-oestradiol) and oestriol. Oestradiol is the most potent and predominant form of oestrogen found in premenopausal women, while oestrone is the most active form of oestrogen in menopausal women and oestriol is the main active form of oestrogen during pregnancy. All three forms of oestrogen are transported in the bloodstream either bound to sex hormone-binding globulin or in the unbound state [23] before binding to either oestrogen receptors (ER) $\alpha$ or $\beta$ expressed on the cell surface of the target tissue [24]. Notably, both oestrogen receptors are expressed in lung epithelia, airway smooth muscle and the pulmonary vasculature $[25,26]$. The activated oestrogen/ER complex has been implicated in fetal alveologenesis and surfactant production, pulmonary arterial hypertension, airway hyperresponsiveness and pulmonary inflammation [25].

Progesterone is likewise produced from cholesterol [27] and readily diffuses across cellular membranes and binds intracellular progesterone receptors A and B. Progesterone receptors are expressed in a number of target tissues, including lung airway epithelium and T-lymphocytes [27-29]. Within the lungs, progesterone has been noted to mediate bronchodilation by reducing contractility and increasing smooth muscle relaxation, confer protection from viral injury and reduce ciliary beat frequency in vitro [28-30].

\section{Menstrual physiology}

In order to understand changes in CF clinical outcomes in the context of female sex hormone fluctuations, the physiological pattern of oestrogen and progesterone change should first be described. While the levels of these hormones are endogenously produced at a higher level in women than in men, oestrogen and progesterone levels fluctuate with monthly menstrual cycles in females of childbearing age. At the start of the cycle, or menstruation, both oestrogen and progesterone levels are low (figure 1). Oestrogen levels subsequently rise and peak, while progesterone levels remain low during the follicular phase (the period of time pre-ovulation) and ovulation. In contrast, during the luteal phase (the period of time after ovulation), both progesterone and oestrogen levels rise before falling again in concert with the start of the next menstrual cycle.

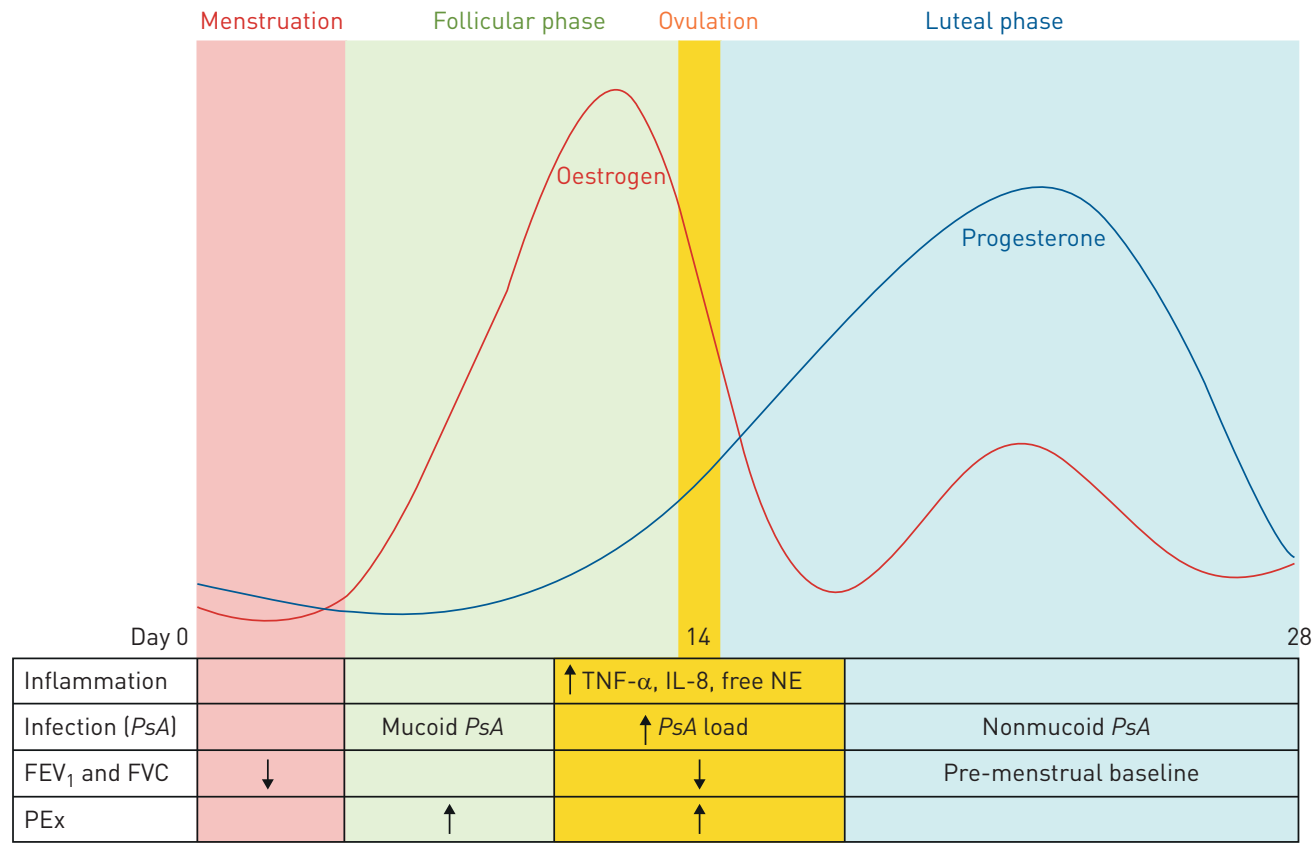

FIGURE 1 Changes in oestrogen and progesterone levels in the context of clinical, infectious and inflammatory findings during the menstrual cycle. During the menstrual phase, both oestrogen and progesterone levels are low. In the follicular phase, oestrogen begins to rise and peak just prior to ovulation. Progesterone begins to rise shortly before ovulation and peak during the luteal phase. Oestrogen levels also increase during the luteal phase. Levels of both progesterone and oestrogen decline until the start of the next menstrual cycle. Forced expiratory volume in $1 \mathrm{~s}\left(\mathrm{FEV}_{1}\right)$ and forced vital capacity (FVC) are both noted to be reduced during menstruation and ovulation but recover to baseline by the luteal phase. Rates of pulmonary exacerbations (PEx) are highest during the follicular phase, while markers of inflammation (tumour necrosis factor- $\alpha$ (TNF- $\alpha$ ), interleukin-8 (IL-8) and free neutrophil elastase (NE)) and Pseudomonas aeruginosa (PsA) sputum bacterial load are highest during ovulation. PSA takes on the more virulent mucoid form during the follicular phase while reverting to the less virulent non-mucoid form during the luteal phase. 


\section{Effects of female sex hormones on CF pathophysiology}

As lung disease is a major determinant of survival in CF, sex differences in airway mechanics may contribute to the "gender gap". The clinical observation of worsening airway infection and inflammation that correspond to increases in the physiological levels of oestrogen and progesterone in women suggest a plausible mechanistic link between sex hormones and CF airway pathophysiology [31].

\section{Impact of sex hormones on mucociliary clearance}

Mucociliary clearance is influenced by a number of factors in the CF patient. The CFTR protein is expressed on the mucosal surface of epithelial cells throughout the body, regulating the secretion and absorption of ions and fluids. Ion transport helps to regulate the $\mathrm{pH}$ and viscosity of airway surface liquid (ASL), a thin fluid layer that sits above the epithelium. Adequate ASL volume is essential for effective mucociliary clearance. In addition, mucus production is a physiological response to the presence of airway debris and microorganisms. CF patients produce more viscous mucus, in part due to CFTR dysfunction, which hampers mucociliary clearance. Suboptimal mucociliary clearance results in retained secretions, mediating an increased vulnerability to recurrent airway infection and inflammation. To understand the link between sex hormones and mucociliary clearance, a number of research groups have examined the effect of oestrogen and progesterone on CFTR expression, CFTR-dependent and CFTR-independent ion transport, ASL and mucus production.

In vitro studies have demonstrated that CFTR expression is either unchanged [32] or even increased in response to oestrogen and/or progesterone exposure [32-34]. In a bronchial epithelial cell line expressing F508del mutated CFTR protein, CASAvOla and colleagues [35] demonstrated that the defect in CFTR localisation and CFTR-mediated chloride secretion could be reversed upon oestrogen stimulation, mediated through the upregulation of the sodium hydrogen exchanger regulatory factor (NHERF1). Similar improvements in CFTR-mediated chloride secretion can be seen in other cell types, such as rabbit pancreatic acinar cells [32]. Conversely, oestrogen appears to have a negative effect on CFTR-independent chloride channel activity. TARRAN and colleagues [36] have demonstrated that 17 - $\beta$-oestradiol can cause increased viscosity of ASL in a dose-dependent fashion by impairing $\mathrm{Ca}^{2+}$-mediated chloride secretion. Similarly, KCNE3 (MinK-related peptide 2) is a protein expressed in tracheal epithelia that regulates KCNQ1 (potassium voltage-gated channel subfamily Q member 1) and has been implicated in the pathophysiology of CF as it indirectly mediates chloride secretion independent of CFTR [37]. Oestrogen has been shown to disrupt the interaction between KCNE3 and KCNQ1, leading indirectly to reduced chloride ion secretion and therefore thicker mucus production [38]. The binding of oestradiol to the oestrogen receptor $\alpha$ in airway epithelial cells has also been shown to increase mucin (MUC5B) production from the submucosal glands, potentially promoting mucus plugging in the CF airways [39]. Finally, oestrogen has also been shown to reduce the intensity of ciliary beat of the CF bronchial epithelium, further hampering the CF airways' ability to clear thickened secretions [40]. Conversely, in non-CF airway epithelium, oestrogen has been shown to increase the intensity of cilia beat frequency and counteracts the negative effects of progesterone on ciliary function [28]. Thus, more work will be needed to understand why oestrogen appears to play opposing roles in $\mathrm{CF}$ and non-CF airways.

Collectively, these findings suggest that while oestrogen may directly improve CFTR expression and function, there are also a number of mechanisms whereby oestrogen can indirectly reduce chloride secretion and thicken mucus/ASL to reduce mucociliary clearance overall.

\section{Impact of sex hormones on immunity and infection}

Female sex hormones, and oestrogen in particular, have been studied in the context of a number of inflammatory, infectious and autoimmune diseases. Depending on the specific arm of the immune response and the disease model, oestrogen has been implicated in both enhancing and suppressing immunity. In general, women tend to have more robust T-cell immunity, which is particularly beneficial in anti-viral defence [41, 42], but exuberant T-helper (Th)-2 response, eosinophilic inflammation and IL-33 production have been implicated in worsening asthma and autoimmune conditions [43, 44]. Conversely, oestrogen appears to hamper the innate immune response to bacterial infections [45]. Sex hormones have also been shown to have pleotropic effects on CF inflammation and vulnerability to bacterial infections. As CF pulmonary exacerbations are frequently mediated by chronic bacterial infections, a sex disparity in bacterial infection response may therefore increase the risk of infection and inflammation in CF women.

The production of lactoferrin, an antimicrobial peptide that is part of the innate defence against invading pathogens, was found to be significantly reduced when oestrogen-stimulated mice were infected with $P$. aeruginosa compared to mice without oestrogen stimulation [46]. McELvaney and colleagues [47] demonstrated that 17 - $\beta$-oestradiol mediates an increase in secretory leukoprotease inhibitor (SLPI) 
production in CF bronchial epithelial cells, resulting in the downregulation of interleukin (IL)-8 production via the Toll-like receptor (TLR)-nuclear factor- $\kappa \mathrm{B}$ signalling pathway. As IL-8 is a chemokine responsible for neutrophil chemotaxis, inhibition of IL-8 production could therefore result in the downregulation of the innate immune response to infections in CF patients. In addition to reduced neutrophilic chemotaxis, oestrogen can also reduce neutrophil-mediated bacterial clearance as neutrophils isolated from oestrogen-treated mice demonstrate increased oxidative burst but reduced $P$. aeruginosa killing when compared to progesterone-treated controls [48]. Oestrogen also appears to promote the Th17 response during $P$. aeruginosa infection as CF mice stimulated with oestrogen demonstrated higher levels of IL-23 and IL-17 in lung tissue and bronchoalveolar fluid when compared to mice without oestrogen stimulation, but despite this, the recovered bacterial load from lung homogenate was paradoxically higher [46]. This suggests that while oestrogen may promote neutrophilic oxidative burst and Th17 response resulting in increased host inflammation and presumably more lung tissue injury, neither response is effective in bacterial clearance. Consistent with this, oestrogen-treated ovariectomised female CF mice were found to have worse survival upon inoculation with $P$. aeruginosa compared to progesterone-treated mice [48].

In addition to modulating the immune response, oestrogen might also directly alter bacterial behaviour. In vitro stimulation with oestradiol was found to enhance the growth of $P$. aeruginosa, promoting the development of the mucoid phenotype via the upregulation of the bacterial mucA gene [49]. Sputum analyses performed during CF patients' menstrual cycle demonstrated a predominance of the mucoid phenotype in the follicular phase (high oestrogen, low progesterone state), while the non-mucoid phenotype predominated during the luteal phase (low oestrogen, high progesterone state) [49]. Mucoid P. aeruginosa is associated with heightened production of alginate, an integral component of biofilm formation. More recently, oestrogen has also been shown to promote virulence factor expression in $P$. aeruginosa isolates, leading to increased $P$. aeruginosa motility as well as increased biofilm formation [40]. Thus, oestrogen may select for a form of $P$. aeruginosa that is more resistant to host or antimicrobial killing.

Little is known about progesterone's effect on infection and inflammation in CF. During pregnancy in non-CF patients, progesterone has been demonstrated to promote Th2 cytokine production while reducing the Th1 cytokine response, playing a critical role in the prevention of fetal rejection by the maternal immune system [50]. In addition, progesterone has been shown to act upon murine pulmonary epithelium to reduce cellular damage and improve lung function post influenza infection by increasing transforming growth factor- $\beta$, IL- 6 and IL-22, as well as inducing the proliferation of CD39 ${ }^{+}$Th17 cells [51]. Progesterone may also be involved in promoting serological responses and T-cell memory, as mice treated with progesterone demonstrate increased antibody titres and lower viral load upon influenza re-infection [52]. Thus, progesterone appears to play an important role in the host immune response to viral infection in murine models, but its role in humans and more specifically CF remains to be seen.

While traditionally considered male sex hormones, androgens are also present in females but at a relatively lower level. "Androgen" is a broad term that encompasses a number of sex hormones including testosterone, dehydroepiandrosterone (DHEA) and DHEA sulfate (DHEA-S) [26]. In non-CF men, testosterone treatment has been shown to be anti-inflammatory, reducing the Th2 response as well as markers of systemic inflammation such as C-reactive protein (CRP) [53]. DHEA-S levels have been demonstrated to inversely correlate with levels of CRP [54]. Several groups have compared androgen levels between CF and non-CF patients. In one small study, serum testosterone levels in five ovulatory CF women were found to be comparable to those in 15 ovulatory non-CF women [55]. More recently, SHAFFER et al. [56] reported lower DHEA-S levels in CF women compared to CF men and non-CF women despite comparable levels between non-CF women and men. It is not clear currently what the clinical implications of the reduced DHEA-S level might be, but this may be a contributing factor to the increased inflammation seen in CF women.

\section{Effects of female sex hormones on pulmonary outcomes}

As oestrogen has been linked to reduced mucociliary clearance and worsening bacterial infection, one could infer that any period of time when oestrogen exposure is heightened there might be increased pulmonary complications and, on the contrary, attenuating the oestrogen surge might mitigate this effect. However, studies examining the relationship between endogenous or exogenous sex hormones and pulmonary outcomes in CF have been conflicting, and therefore the relationship is likely to be much more complicated (table 1).

\section{Endogenous changes}

At clinical baseline, women with CF have comparable levels of serum oestradiol to non-CF women [57]. Interestingly, СнотіRmall and colleagues [49] found that during periods of CF exacerbations, serum oestradiol levels are significantly higher. They also demonstrated a correlation between serum levels of oestradiol and the frequency of infectious pulmonary exacerbations where the majority of events occurred 
TABLE 1 Summary of animal and human studies in cystic fibrosis (CF) examining the effects of female sex hormones or cystic fibrosis transmembrane conductance regulator (CFTR) modulators

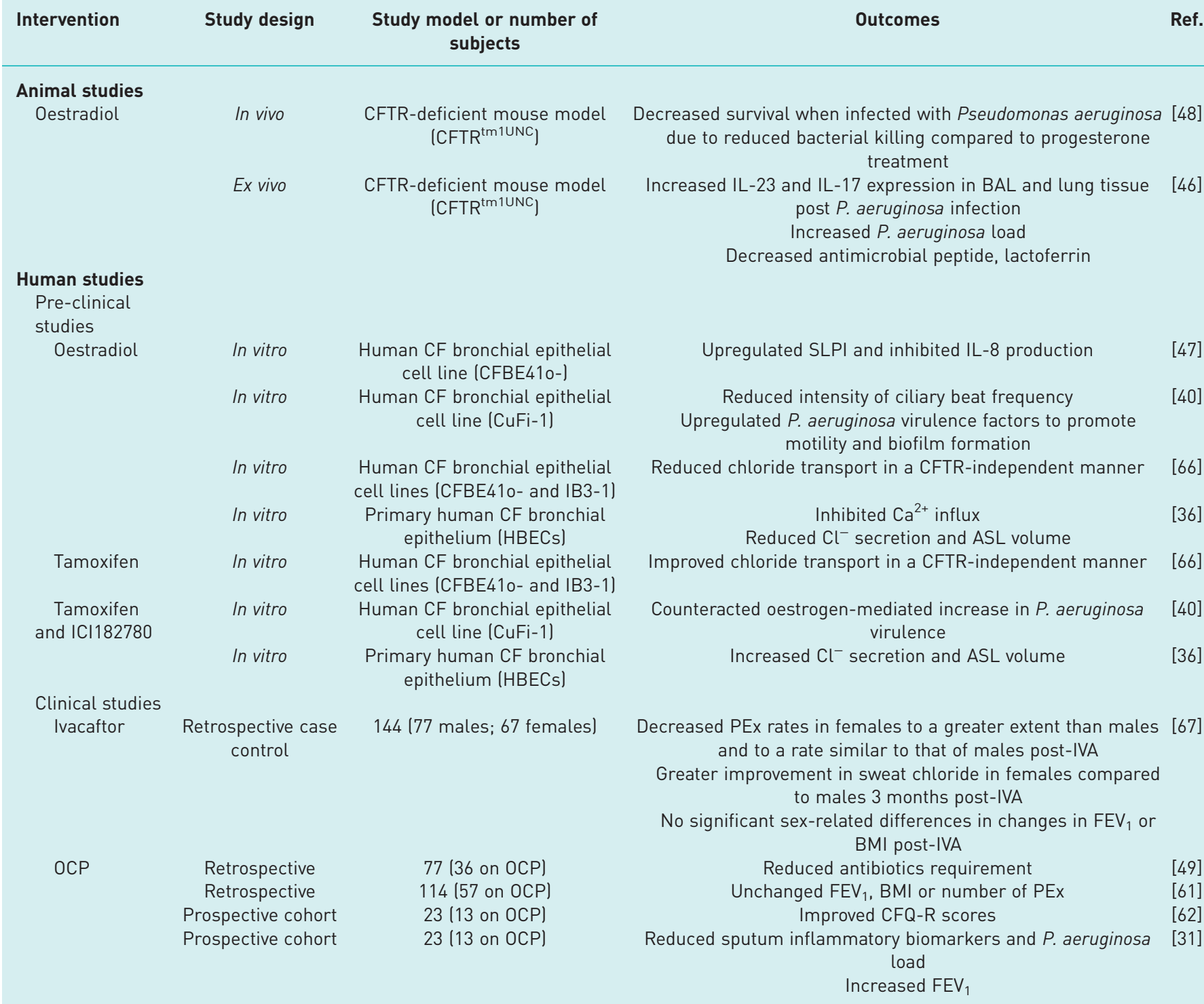

IL: interleukin; BAL: bronchoalveolar lavage; SLPI: secretory leukoprotease inhibitor; ASL: airway surface liquid; PEx: pulmonary exacerbation; IVA: ivacaftor; OCP: oral contraceptive pill; $\mathrm{FEV}_{1}$ : forced expiratory volume in $1 \mathrm{~s}$; BMI: body mass index; CFQ-R: cystic fibrosis questionnaire revised.

during the follicular phase (high oestrogen, low progesterone) [49]. Furthermore, registry data collected during pregnancy, a period of time where oestrogen and progesterone are both heightened (table 2), suggest that annual rates of pulmonary exacerbations [58] and haemoptysis [59] are higher compared to non-pregnant female controls. However, it should be noted that the increased rates of exacerbations found during pregnancy might simply be a reflection of increased frequency and intensity of monitoring or other non-hormone related physiological changes in pregnancy (such as increased acid reflux) that heighten the risk of pulmonary complications. Nevertheless, these observations support the hypothesis that elevations in oestrogen may have a detrimental effect on pulmonary infections and presumably lung function. However, when JOHANNESSON and colleagues [60] monitored $12 \mathrm{CF}$ women during their menstrual cycles, they found a $3-5 \%$ reduction in both forced expiratory volume in $1 \mathrm{~s}\left(\mathrm{FEV}_{1}\right)$ and forced vital capacity during menstruation (low oestrogen and progesterone) and ovulation (high oestrogen, low progesterone), which recovered during the luteal phase despite representing a period of high oestrogen and progesterone. Thus, the physiological changes in female sex hormones may have a more complex relationship with fluctuations in lung function and mucociliary clearance. 
TABLE 2 Serum concentrations of oestradiol and progesterone during the menstrual cycle, pregnancy and with oral contraceptive pill (OCP) use

Serum oestradiol $\mathrm{pg} \cdot \mathrm{mL}^{-1}$

Menstruation
Follicular phase
Ovulation
Luteal phase
Pregnancy (singleton; peak level)
OCP (monophasic at steady state)"
Men

\#: during pregnancy, oestrogen and progesterone levels rise steadily throughout the trimesters; ranges provided in table represent peak levels documented in the third trimester. ": ranges of serum oestradiol and progesterone reflect steady-state serum concentrations achieved after multiple months of use with monophasic OCP from products with the lowest to the highest oestradiol/progesterone concentration.

\section{Exogenous changes}

The most commonly used hormone-based combination oral contraceptive pills (OCPs) works by inhibiting ovulation by providing 21 days of low-dose oestradiol and progesterone each month to abrogate the natural rise and fall of oestrogen and progesterone during the menstrual cycle (figure 1). Based on a large retrospective study involving 700 women with CF followed over 5 years, no statistically significant difference with regards to the annual rates of change in $\mathrm{FEV}_{1}$ or in the numbers of pulmonary exacerbations requiring intravenous antibiotics were observed between women using OCPs compared to those who did not [61]. Paired analysis of the same women pre- and post-OCP over 3 years also failed to show any difference in clinical outcomes. A separate retrospective analysis involving the Irish CF registry also did not find a statistically significant difference in outcomes in women using OCPs [49]. However, a trend towards reduction in the number of pulmonary exacerbations requiring treatment with either oral or intravenous antibiotics was seen with longer term OCP use [49]. While such retrospective registry studies can be helpful in identifying possible trends, it should be pointed out that there are a number of OCP products on the market that have different combined concentrations of oestrogen and progesterone or are oestrogen/progesterone monotherapies. As well, OCPs are categorised into monophasic (same dose for 21 days of the month) and multiphasic (escalation in dose throughout the month) products. Consequently, without capturing the details of the type of OCP used, potential effects of oestrogen and progesterone on pulmonary outcomes can be challenging to study in observational studies.

A recent small prospective study presented in abstract form by JAIN and colleagues [31] demonstrated that $\mathrm{FEV}_{1}$ increased by $2.5 \%$ in women who were started on the OCP when compared to their pre-OCP ovulation phase $\mathrm{FEV}_{1}$. Clinically, assessment of patient reported symptom score using the CF questionnaire - revised (CFQ-R) as well as other patient reported quality of life scores demonstrated clinically significant improvements once the OCP was initiated [62]. Furthermore, prior to OCP initiation, these scores were found to be lower in women during menstruation and ovulation when compared to men. Biochemically, sputum inflammatory markers (including tumour necrosis factor- $\alpha$, IL- 8 and free neutrophil elastase) and $P$. aeruginosa bacterial load were higher during ovulation than in the menstrual or luteal phases [31]. Importantly, a significant reduction in these sputum bacterial and biomarker findings were seen once the OCP was initiated. Given that the OCP typically provides a consistently lower serum concentration of oestrogen and higher concentration of progesterone than the physiological rise and fall of either sex hormones (figure 1), these clinical observations may suggest that reduction in inflammatory activity and bacterial load may result from either a higher level of progesterone, a combination of lower steady state of oestrogen and higher steady state of progesterone, or the abrogation of physiological hormonal fluctuations.

There has been a rise in the number of individuals identifying as transgender in the USA over the past decade. Hormone therapies are commonly employed at the initial stage of gender transitioning from male to female to target a serum oestradiol level in the range of $100-200 \mathrm{ng} \cdot \mathrm{mL}^{-1}$, similar to that seen during ovulation (table 2). While there is currently no published data on how hormone therapies might influence CF relevant pulmonary outcomes, there is an increased lifetime risk of developing asthma in transgender females (male-to-female) relative to transgender males (female-to-male) or non-transgender individuals [63]. In our centre, we currently have a patient who is receiving hormone therapy as part of sex reassignment. Anecdotally, she experienced persistent drops in $\mathrm{FEV}_{1}$ with increased frequency of 
pulmonary exacerbations, coinciding with oestrogen dose escalation [64]. Similarly, for women experiencing uncomfortable symptoms of menopause, oestrogen and/or progesterone is often prescribed as hormone replacement therapy (HRT) for symptomatic relief. While there is no literature to date on the effects of HRT in the CF population, a prospective analysis involving non-CF individuals examining lung disease outcomes found an increased rate of adult onset asthma with oestrogen only or combination oestrogen/progesterone HRT use [65].

\section{Therapeutic targeting of female sex hormones}

In vitro and in vivo studies have demonstrated that oestrogen receptor antagonism may be effective at reversing the oestrogen-mediated pro-inflammatory effects seen in CF airways. Tamoxifen, a selective oestrogen receptor modulator that has both anti-oestrogenic and pro-oestrogenic effects depending on the target tissue, and other non-clinically available oestrogen antagonists have been shown to counteract oestrogen-mediated increases in P. aeruginosa virulence [40]. Tamoxifen has also been shown to improve chloride transport in CF epithelial cells in a CFTR-independent manner by increasing calcium-activated chloride channel currents [66]. While modulating oestrogen levels or oestrogen receptor antagonism has the potential to reduce inflammation and improve pulmonary outcomes in CF females, oestrogen has a wide range of physiological effects including the regulation of menstrual cycles and fertility, bone health and the development of secondary sex characteristics. Therefore, a more targeted approach to altering the downstream effect of oestrogen-mediated inflammation is likely to be a more prudent strategy. In addition, it has been observed that the disparity between men and women with regards to pulmonary exacerbation rates can be improved or even abolished in women treated with CFTR modulators such as ivacaftor [67] or lumacaftor-ivacaftor [68], suggesting that improvements in CFTR expression, localisation or function might be sufficient to counteract the negative effects of oestrogen on CFTR-independent pathways, infection, inflammation and mucociliary clearance.

Unlike oestrogen, much less attention has been paid towards the potential therapeutic benefits of progesterone treatment. Studies of female physiology during pregnancy demonstrate that progesterone has a bronchodilatory effect on the airways through relaxation of smooth muscle tone [69]. Thus, therapeutic dosing of progesterone to mimic levels achieved in pregnancy (table 2) might be a viable intervention. As the progesterone-only OCP is much less popular than the combined OCP due to the concern about accelerated bone loss with both short- and long-term use [70,71], there is little observational data on the effect of progesterone alone. Given that the therapeutic concentration of progesterone provided in the combined OCP is higher than the physiological level of progesterone during ovulation (table 2), the reduction in sputum inflammation seen with OCP use [31] might be attributable to higher progesterone levels alone. Alternatively, given that the OCP provides a steady-state concentration of progesterone, eliminating the typical rise and fall of sex hormones throughout the menstrual cycle, the improvement in sputum inflammation could also be due to the abrogation of large swings in sex hormone levels. Consequently, further understanding of the effect of progesterone on human lung inflammation will be important before considering the therapeutic use of progesterone in this context.

\section{Future research}

The study of sex hormones and CF holds much potential not only for furthering our understanding of inflammation and infection in CF host-pathogen interactions but also for the design of novel therapeutics to help reduce pulmonary-related morbidity and mortality. Several key areas warrant further investigation to achieve these goals. First, further research is needed to understand the mechanistic role that progesterone and the complex interaction between oestrogen and progesterone stimulation might have on mucociliary clearance and bacterial response. While in vitro work demonstrates that oestrogen alone is pro-inflammatory and promotes bacterial survival, clinical observations of improved pulmonary outcomes during the luteal phase (high oestrogen and progesterone) when compared to the follicular phase or ovulation (both high oestrogen and low progesterone states) suggest that the mere presence of high levels of oestrogen does not always result in worse pulmonary outcomes. Given that oestrogen and progesterone levels are similar in men and menstruating women, the fluctuations or surges in sex hormone levels during the female menstrual cycle might be more important than the absolute level of the hormones themselves. Thus, the effect of progesterone and the cyclical changes in oestrogen and progesterone exposure to CF airways merits further investigation. Second, as sex hormones have a myriad of endocrinological effects, oestrogen and progesterone-based therapy may not be a viable intervention to reduce pulmonary exacerbations. Therefore, further research should be focused on finding downstream components of the pro-inflammatory pathway mediated by oestrogen receptor activation that might be a more specific target for therapeutic intervention, such as the SLPI. Further mechanistic studies should be directed to better understand the oestrogen receptor pathway in bronchial epithelial cells to look for other specific localised targets without causing other unintended endocrinological effects. 


\section{Conclusion}

While it currently remains unclear why a sex disparity exists in CF survival, studies performed over the last several decades have provided evidence that sex hormones might contribute to poor pulmonary outcomes in females by impairing mucociliary clearance and the immune response to infection, increasing bacterial virulence and increasing lung inflammation. Additional research is needed to clarify the role of progesterone on CF airways and to identify specific treatments that counteract the negative effects of female sex hormones on CF airway pathophysiology to improve outcomes in women with CF without causing broader endocrinological complications.

Author contributions: G.Y. Lam: conceptualisation, funding acquisition and writing - original draft. J. Goodwin and P.G. Wilcox: writing - review and editing. B.S. Quon: supervision, funding acquisition and writing - review and editing. All authors read and approved the manuscript.

Conflict of interest: G.Y. Lam has nothing to disclose. J. Goodwin has nothing to disclose. P.G. Wilcox has nothing to disclose. B.S. Quon reports speaker fees from Vertex Pharmaceuticals, advisory board fees from Proteostasis Therapeutics, and grants from CF Canada, the CF Foundation, Gilead Sciences and the BC Lung Association, outside the submitted work.

Support statement: A CF Canada Gilead Sciences Fellowship Award (G.Y. Lam) and a Michael Smith Foundation for Health Research Scholar award (B.S. Quon) provided funding for clinician research time to prepare this manuscript. Funding information for this article has been deposited with the Crossref Funder Registry.

\section{References}

1 McColley SA, Schechter MS, Morgan WJ, et al. Risk factors for mortality before age 18 years in cystic fibrosis. Pediatr Pulmonol 2017; 52: 909-915.

2 Kerem E, Reisman J, Corey M, et al. Prediction of mortality in patients with cystic fibrosis. N Engl J Med 1992; 326: $1187-1191$.

3 Raghavan D, Jain R. Increasing awareness of sex differences in airway diseases. Respirology 2016; 21: 449-459.

4 LoMauro A, Aliverti A. Sex differences in respiratory function. Breathe (Sheff) 2018; 14: 131-140.

5 Rosenfeld M, Davis R, FitzSimmons S, et al. Gender gap in cystic fibrosis mortality. Am J Epidemiol 1997; 145 794-803.

6 Cystic Fibrosis Canada. The Canadian Cystic Fibrosis Registry. 2017 Annual Data Report. www.cysticfibrosis.ca/ blog/2017-registry-annual-data-report/. Date last accessed: July 2020. Date last updated: December 2018.

7 Reid DW, Blizzard CL, Shugg DM, et al. Changes in cystic fibrosis mortality in Australia, 1979-2005. Med J Aust 2011; 195: 392-395.

8 Olesen HV, Pressler T, Hjelte L, et al. Scandinavian Cystic Fibrosis Study Consortium. Gender differences in the Scandinavian cystic fibrosis population. Pediatr Pulmonol 2010; 45: 959-965.

9 Harness-Brumley CL, Elliott AC, Rosenbluth DB, et al. Gender differences in outcomes of patients with cystic fibrosis. J Womens Health (Larchmt) 2014; 23: 1012-1020.

10 Cystic Fibrosis Trust. UK Cystic Fibrosis Registry: Annual Data Report 2017. Date last accessed: July 2020. Date last updated: August 2018. https://www.cysticfibrosis.org.uk/ /media/documents/the-work-wedo/uk-cf-registry/ 2017-registry-annual-data-report-updated-291018.ashx?la=en

11 de Jong CCM, Slabbers L, Engel TGP, et al. Clinical relevance of Scedosporium spp. and Exophiala dermatitidis in patients with cystic fibrosis: a nationwide study. Med Mycol 2020; 58: 859-866.

12 Miller RJ, Tildesley HD, Wilcox PG, et al. Sex disparities in effects of cystic fibrosis-related diabetes on clinical outcomes: a matched study. Can Respir J 2008; 15: 291-294.

13 Stephenson AL, Tom M, Berthiaume Y, et al. A contemporary survival analysis of individuals with cystic fibrosis: a cohort study. Eur Respir J 2015; 45: 670-679.

14 Boelle PY, Viviani L, Busson PF, et al. Reference percentiles for FEV(1) and BMI in European children and adults with cystic fibrosis. Orphanet J Rare Dis 2012; 7: 64-64.

15 Simon SL, Duncan CL, Horky SC, et al. Body satisfaction, nutritional adherence, and quality of life in youth with cystic fibrosis. Pediatr Pulmonol 2011; 46: 1085-1092.

16 Zemel BS, Jawad AF, FitzSimmons S, et al. Longitudinal relationship among growth, nutritional status, and pulmonary function in children with cystic fibrosis: analysis of the Cystic Fibrosis Foundation National CF Patient Registry. J Pediatr 2000; 137: 374-380.

17 Patterson JM, Wall M, Berge J, et al. Associations of psychosocial factors with health outcomes among youth with cystic fibrosis. Pediatr Pulmonol 2009; 44: 46-53.

18 Simon MISDS, Paulo GCF, Marostica JC. Body mass index and albumin levels are associated with pulmonary function parameters in pediatric subjects with cystic fibrosis. Rev Paul Pediatr 2019; 37: 414-418.

19 Patterson JM, Wall M, Berge J, et al. Gender differences in treatment adherence among youth with cystic fibrosis: development of a new questionnaire. J Cyst Fibros 2008; 7: 154-164.

20 Viviani L, Bossi A, Assael BM, et al. Absence of a gender gap in survival. An analysis of the Italian registry for cystic fibrosis in the paediatric age. J Cyst Fibros 2011; 10: 313-317.

21 Sutton S, Rosenbluth D, Raghavan D, et al. Effects of puberty on cystic fibrosis related pulmonary exacerbations in women versus men. Pediatr Pulmonol 2014; 49: 28-35.

22 Cui J, Shen Y, Li R. Estrogen synthesis and signaling pathways during aging: from periphery to brain. Trends Mol Med 2013; 19: 197-209.

23 Laurent MR, Hammond GL, Blokland M, et al. Sex hormone-binding globulin regulation of androgen bioactivity in vivo: validation of the free hormone hypothesis. Sci Rep 2016; 6: 35539.

24 Hamilton KJ, Hewitt SC, Arao Y, et al. Estrogen hormone biology. Curr Top Dev Biol 2017; 125: 109-146. 
Assaggaf H, Felty Q. Gender, estrogen, and obliterative lesions in the lung. Int J Endocrinol 2017; 2017: 8475701. Sathish V, Martin YN, Prakash YS. Sex steroid signaling: implications for lung diseases. Pharmacol Ther 2015; 150: $94-108$

Taraborrelli S. Physiology, production and action of progesterone. Acta Obstet Gynecol Scand 2015; 94: Suppl. 161, 8-16.

Jain R, Ray JM, Pan JH, et al. Sex hormone-dependent regulation of cilia beat frequency in airway epithelium. Am J Respir Cell Mol Biol 2012; 46: 446-453.

Perusquia M, Hernandez R, Montano LM, et al. Inhibitory effect of sex steroids on guinea-pig airway smooth muscle contractions. Comp Biochem Physiol C Pharmacol Toxicol Endocrinol 1997; 118: 5-10.

Klein SL, Flanagan KL. Sex differences in immune responses. Nat Rev Immunol 2016; 16: 626-638.

Jain R, Sabanova V, Keller A, et al. Effects of sex hormones on markers of health in women with cystic fibrosis. Pediatr Pulmonol 2019; 54: 221.

pancreatic epithelial cells. Am J Physiol 1996; 271 : 747.

Rochwerger L, Buchwald M. Stimulation of the cystic fibrosis transmembrane regulator expression by estrogen in vivo. Endocrinology 1993; 133: 921-930.

Ajonuma LC, Tsang LL, Zhang GH, et al. Estrogen-induced abnormally high cystic fibrosis transmembrane conductance regulator expression results in ovarian hyperstimulation syndrome. Mol Endocrinol 2005; 19: 3038-3044.

Fanelli T, Cardone RA, Favia M, et al. Beta-oestradiol rescues DeltaF508CFTR functional expression in human cystic fibrosis airway CFBE41o- cells through the up-regulation of NHERF1. Biol Cell 2008; 100: 399-412.

Coakley RD, Sun H, Clunes LA, et al. 17beta-Estradiol inhibits Ca2+-dependent homeostasis of airway surface liquid volume in human cystic fibrosis airway epithelia. J Clin Invest 2008; 118: 4025-4035.

Preston P, Wartosch L, Gunzel D, et al. Disruption of the K+ channel beta-subunit KCNE3 reveals an important role in intestinal and tracheal Cl- transport. J Biol Chem 2010; 285: 7165-7175.

Kroncke BM, Van Horn WD, Smith J, et al. Structural basis for KCNE3 modulation of potassium recycling in epithelia. Sci Adv 2016; 2: e1501228.

Choi HJ, Chung YS, Kim HJ, et al. Signal pathway of 17beta-estradiol-induced MUC5B expression in human airway epithelial cells. Am J Respir Cell Mol Biol 2009; 40: 168-178.

Tyrrell J, Harvey BJ. Sexual dimorphism in the microbiology of the CF 'Gender Gap': estrogen modulation of Pseudomonas aeruginosa virulence. Steroids 2020; 156: 108575.

Robinson DP, Hall OJ, Nilles TL, et al. 17beta-estradiol protects females against influenza by recruiting neutrophils and increasing virus-specific CD8 T cell responses in the lungs. J Virol 2014; 88: 4711-4720.

Takahashi T, Ellingson MK, Wong $\mathrm{P}$, et al. Sex differences in immune responses that underlie COVID-19 disease outcomes. Nature 2020; 588: 315-320.

Desai MK, Brinton RD. Autoimmune disease in women: endocrine transition and risk across the lifespan. Front Endocrinol (Lausanne) 2019; 10: 265.

4 Watanabe Y, Tajiki-Nishino R, Tajima H, et al. Role of estrogen receptors alpha and beta in the development of allergic airway inflammation in mice: a possible involvement of interleukin 33 and eosinophils. Toxicology 2019; 411: $93-100$

Speyer CL, Rancilio NJ, McClintock SD, et al. Regulatory effects of estrogen on acute lung inflammation in mice. Am J Physiol Cell Physiol 2005; 288: 881.

Wang Y, Cela E, Gagnon S, et al. Estrogen aggravates inflammation in Pseudomonas aeruginosa pneumonia in cystic fibrosis mice. Respir Res 2010; 11: 166-166.

Chotirmall SH, Greene CM, Oglesby IK, et al. 17Beta-estradiol inhibits IL-8 in cystic fibrosis by up-regulating secretory leucoprotease inhibitor. Am J Respir Crit Care Med 2010; 182: 62-72.

Abid S, Xie S, Bose M, et al. 17beta-Estradiol dysregulates innate immune responses to Pseudomonas aeruginosa respiratory infection and is modulated by estrogen receptor antagonism. Infect Immun 2017; 85: e0422-17.

Chotirmall SH, Smith SG, Gunaratnam C, et al. Effect of estrogen on pseudomonas mucoidy and exacerbations in cystic fibrosis. N Engl J Med 2012; 366: 1978-1986.

Kumar P, Magon N. Hormones in pregnancy. Niger Med J 2012; 53: 179-183.

Hall OJ, Limjunyawong N, Vermillion MS, et al. Progesterone-based therapy protects against influenza by promoting lung repair and recovery in females. PLoS Pathog 2016; 12: e1005840.

Hall OJ, Nachbagauer R, Vermillion MS, et al. Progesterone-based contraceptives reduce adaptive immune responses and protection against sequential Influenza A virus infections. J Virol 2017; 91: e02160-16.

Bianchi VE. The anti-inflammatory effects of testosterone. J Endocr Soc 2019; 3: 91-107.

Haring R, Baumeister SE, Volzke H, et al. Prospective inverse associations of sex hormone concentrations in men with biomarkers of inflammation and oxidative stress. J Androl 2012; 33: 944-950.

5 Johannesson M, Landgren BM, Csemiczky G, et al. Female patients with cystic fibrosis suffer from reproductive endocrinological disorders despite good clinical status. Hum Reprod 1998; 13: 2092-2097.

6 Shaffer L, Abid S, Jain R. Evaluation of dehydroepiandrosterone sulfate (DHEA-S) levels in cystic fibrosis. Am J Respir Crit Care Med 2019; 199: A6178.

7 Schram CA, Stephenson AL, Hannam TG, et al. Cystic fibrosis (CF) and ovarian reserve: a cross-sectional study examining serum anti-mullerian hormone (AMH) in young women. J Cyst Fibros 2015; 14: 398-402.

5 McMullen AH, Pasta DJ, Frederick PD, et al. Impact of pregnancy on women with cystic fibrosis. Chest 2006; 129: 706-711.

9 Renton M, Priestley L, Bennett L, et al. Pregnancy outcomes in cystic fibrosis: a 10-year experience from a UK centre. Obstet Med 2015; 8: 99-101.

Johannesson M, Ludviksdottir D, Janson C. Lung function changes in relation to menstrual cycle in females with cystic fibrosis. Respir Med 2000; 94: 1043-1046.

Kernan NG, Alton EW, Cullinan P, et al. Oral contraceptives do not appear to affect cystic fibrosis disease severity. Eur Respir J 2013; 41: 67-73.

Jain R, Sabanova V, Holtrop M, et al. Symptom differences and health perceptions between men and women with cystic fibrosis. Pediatr Pulmonol 2019; 54: 424. 
63 Morales-Estrella JL, Boyle M, Zein JG. Transgender status is associated with higher risk of lifetime asthma. Am J Respir Crit Care Med 2018; 197: A34.

64 Lam GY, Goodwin J, Wilcox P, et al. Worsening pulmonary outcomes during sex reassignment therapy in a transgender female with cystic fibrosis (CF) and asthma/allergic bronchopulmonary aspergillosis: a case report. BMC Pulm Med 2020; 20: 234.

65 Barr RG, Wentowski CC, Grodstein F, et al. Prospective study of postmenopausal hormone use and newly diagnosed asthma and chronic obstructive pulmonary disease. Arch Intern Med 2004; 164: 379-386.

66 Imberti R, Garavaglia ML, Verduci I, et al. Antiestrogen- and tamoxifen-induced effects on calcium-activated chloride currents in epithelial cells carrying the F508-CFTR point mutation. Respir Res 2018; 19: 198-191.

67 Secunda KE, Guimbellot JS, Jovanovic B, et al. Females with cystic fibrosis demonstrate a differential response profile to Ivacaftor compared to males. Am J Respir Crit Care Med 2020; 201: 996-998.

68 Aalbers BL, Hofland RW, Bronsveld I, et al. Females with cystic fibrosis have a larger decrease in sweat chloride in response to lumacaftor/ivacaftor compared to males. J Cyst Fibros 2020; in press [https://doi.org/10.1016/j.jcf.2020. 05.004].

69 LoMauro A, Aliverti A. Respiratory physiology of pregnancy: physiology masterclass. Breathe (Sheff) 2015; 11: 297-301.

70 Cundy T, Cornish J, Roberts $\mathrm{H}$, et al. Spinal bone density in women using depot medroxyprogesterone contraception. Obstet Gynecol 1998; 92: 569-573.

71 Petitti DB, Piaggio G, Mehta S, et al. Steroid hormone contraception and bone mineral density: a cross-sectional study in an international population. The WHO Study of Hormonal Contraception and Bone Health. Obstet Gynecol 2000; 95: 736-744. 